\title{
HIV-1 Integrase Strand Transfer Inhibitors with Reduced Susceptibility to Drug Resistant Mutant Integrases
}

Xue Zhi Zhao, ${ }^{\dagger}$ Steven J. Smith, ${ }^{\ddagger}$ Daniel P. Maskell, " Mathieu Metifiot, ${ }^{\S}$ Valerie E. Pye, ${ }^{\|}$Katherine Fesen, ${ }^{\S}$ Christophe Marchand, ${ }^{\S}$ Yves Pommier, ${ }^{\S}$ Peter Cherepanov, ${ }^{\|, \perp}$ Stephen H. Hughes, ${ }^{\S}$ and Terrence R. Burke, Jr.* ${ }^{\dagger}$

${ }^{\dagger}$ Chemical Biology Laboratory and ${ }^{\star}$ HIV Dynamics and Replication Program, Center for Cancer Research, National Cancer Institute, National Institutes of Health, Frederick, Maryland 21702, United States

${ }^{\S}$ Developmental Therapeutics Branch and Laboratory of Molecular Pharmacology, Center for Cancer Research, National Cancer Institute, National Institutes of Health, Bethesda, Maryland 20892, United States

"Clare Hall Laboratories, The Francis Crick Institute, Blanche Lane, South Mimms, EN6 3LD, United Kingdom

${ }^{\perp}$ Imperial College London, St-Mary's Campus, Norfolk Place, London, W2 1PG, United Kingdom

\section{Supporting Information}

ABSTRACT: HIV integrase (IN) strand transfer inhibitors (INSTIs) are among the newest anti-AIDS drugs; however, mutant forms of IN can confer resistance. We developed noncytotoxic naphthyridine-containing INSTIs that retain low nanomolar $\mathrm{IC}_{50}$ values against HIV-1 variants harboring all of the major INSTI-resistant mutations. We found by analyzing crystal structures of inhibitors bound to the IN from the prototype foamy virus (PFV) that the most successful inhibitors show striking mimicry of the bound viral DNA prior to $3^{\prime}$-processing and the bound host DNA prior to strand transfer. Using this concept of "bi-substrate mimicry," we developed a new broadly effective

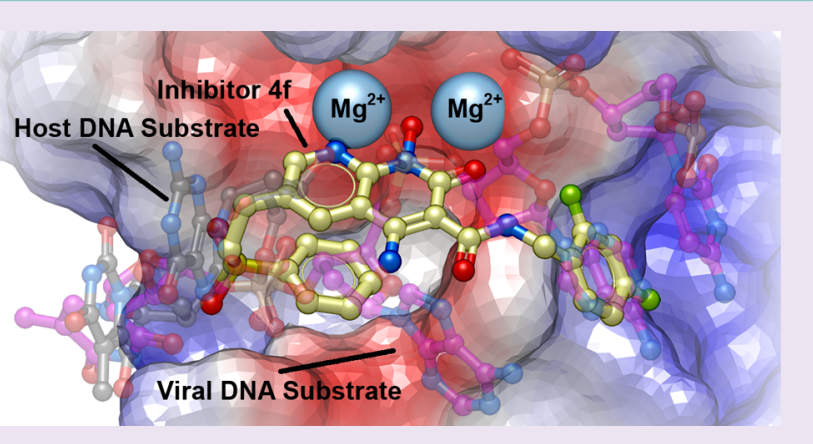
inhibitor that not only mimics aspects of both the bound target and viral DNA but also more completely fills the space they would normally occupy. Maximizing shape complementarity and recapitulating structural components encompassing both of the IN DNA substrates could serve as a guiding principle for the development of new INSTIs.

\begin{abstract}
Thibiting enzymes that modify DNA or RNA substrates has the potential to be used in the treatment of a wide range of diseases. Polynucleotide transferases are important targets for drug development; however, the catalytic centers of these enzymes are often extended to accommodate their large substrates. It has been difficult to design potent, specific, and selective small molecule inhibitors. Fortunately, this has not been an intractable problem, as exemplified by the development of drugs that act as interfacial inhibitors of HIV-1 integrase (IN), ${ }^{1}$ one of three viral enzymes that are essential for viral replication. IN inserts viral DNA into the host genome through two sequential reactions. The first reaction, termed " 3 'processing" ( $\left.3^{\prime}-\mathrm{P}\right)$, involves the cleavage of the $3^{\prime}$-dinucleotides from viral DNA; the second reaction "strand transfer" (ST) ${ }^{2}$ involves the subsequent insertion of the processed ends of the viral DNA into host DNA. These reactions take place in the context of a stable nucleoprotein complex containing a multimer of IN assembled on viral DNA ends, referred to as the intasome. Divalent metal cofactors are essential for INmediated catalysis, and chelation of the active site $\mathrm{Mg}^{2+}$ ions is a key component of the successful integrase strand transfer inhibitors (INSTIs), which represent the newest class of anti-
\end{abstract}

AIDS drugs. ${ }^{3}$ However, in infected patients, the HIV-1 genome diversifies rapidly due to an accumulation of mutations that arise during viral replication. ${ }^{4,5}$ Consequently, the emergence of resistance is a limitation for all anti-HIV therapeutics, including INSTIs, and resistant forms of the virus have been isolated from patients who received raltegravir (RAL, 1) or elvitegravir (EVG, 2), the first two INSTIs approved by the FDA for the treatment of HIV/AIDS.,6,7 More recently, dolutegravir (DTG, 3) has come to market as a second generation INSTI. DTG appears to be considerably less prone to virological failure than RAL and EVG. ${ }^{8-12}$ However, viruses that carry IN resistance mutations can be selected by growing the virus in the presence of DTG, and mutations that confer cross-resistance to all three INSTIs have been identified. ${ }^{13-17}$

A primary objective of continued INSTI development is to discover compounds with minimal toxicity that retain good effectiveness against existing resistant mutants. ${ }^{13}$ Until now, efforts to create new drugs that retain broad efficacy against the

Received: November 17, 2015

Accepted: January 25, 2016

Published: January 25, 2016 
resistant forms of IN have largely been empirical. This is in spite of the availability of "cocrystal" structures of the leading first and second-generation INSTIs bound to the intasome formed by the othologous enzyme from prototype foamy virus (PFV) in a complex with metal cofactors and the cognate viral DNA substrate (referred to as the "intasome"). ${ }^{18-21}$ Although these advances elucidated the structural basis for INSTI function, they have not yet led to principles that can guide the design of the next generation INSTIs, which would retain effectiveness against the known resistant IN mutants. ${ }^{16,22}$

In our current work, we empirically varied the structure of our recently disclosed 1-hydroxy-2-oxo-1,8-naphthyridine-containing INSTIs (4) to improve their ability to potently block in cell culture, the replication of a panel of HIV-1 based vectors that carry all of the major INSTI-resistant IN mutants. ${ }^{23,24}$ We also obtained "cocrystal" structures of the PFV intasome with the best inhibitors and found that when they bind to IN, they show striking mimicry of the binding of viral DNA in its initial state, prior to the cleavage of the dinucleotide in the $3^{\prime}-\mathrm{P}$ reaction. The inhibitors also mimic features of target DNA in their binding to the intasome active site. ${ }^{25}$ Our findings suggest that employing aspects of molecular mimicry of viral and host DNA may be useful in the design of improved INSTIs. Importantly, these principles may also be applicable to the broader class of enzymes that have DNA or RNA substrates.

\section{RESULTS AND DISCUSSION}

Development of INSTIs That Retain Efficacy against RAL-resistant Mutant Forms of IN. INSTIs selectively bind at the interface of IN and the viral DNA end following its $3^{\prime}$-P. ${ }^{18,19,25}$ INSTIs contain a triad of heteroatoms (shown in red in Figure 1) that chelate the essential pair of $\mathrm{Mg}^{2+}$ ions in
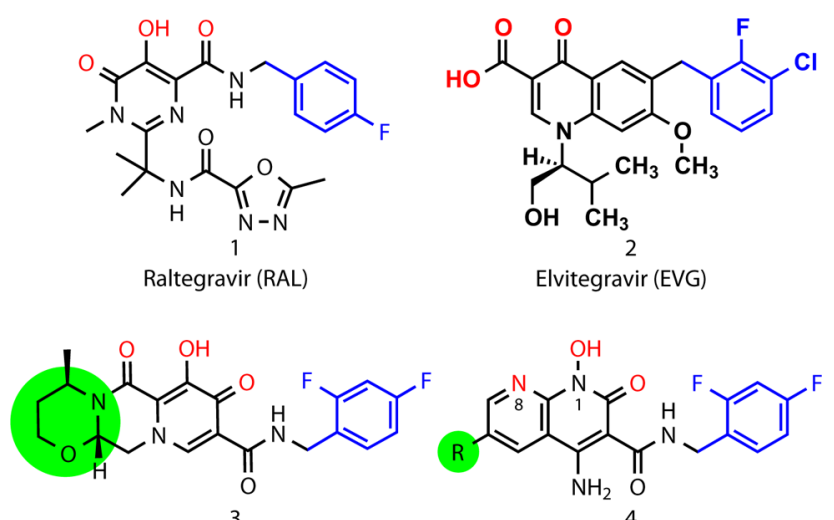

Dolutegravir (DTG)

Figure 1. Structures of the FDA-approved INSTIs (RAL, 1; EVG, 2; and DTG, 3) and heterobicyclic compounds of the current series (4). Colors highlight key functional features: metal-chelating triad of heteroatoms (red), halobenzyl rings (blue). The terminal ring of DTG and the corresponding 6-substituents described in the present work shown in green shading.

the IN active site. INSTIs also typically include a halobenzyl ring that stacks against the base of the penultimate deoxycytidine near the processed 3 '-end of the viral DNA (shown in blue in Figure 1). ${ }^{3,26}$ Our long-term goal is to develop small molecules that are active against IN mutants that are resistant to current INSTIs. Initially, our efforts were directed at the retention of efficacy against the Y143R and $\mathrm{N} 155 \mathrm{H}$ mutants and the double mutant, Q148H/G140S. ${ }^{13-16}$
While these are associated with clinical HIV-1 resistance to $\mathrm{RAL}$, there is a considerable overlap in the resistance profiles of RAL and EVG. ${ }^{27,28}$ Prior to the current work, we had performed extensive investigations on a core 1-hydroxy-2-oxo1,8-naphthyridine platform. ${ }^{23,24}$ These efforts yielded compound $4 \mathrm{a}$, with a ST inhibitory value of $\mathrm{IC}_{50}=19 \mathrm{nM}$ in an in vitro IN assay (compound $\mathbf{4 a}$, Table 1 ) and an antiviral potency

Table 1. Inhibitory Potencies of Compounds 4a-4f Determined Using an in Vitro IN Assay ${ }^{a}$

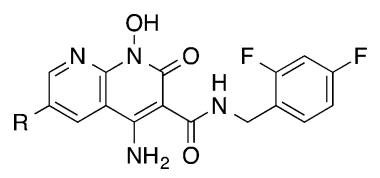

\begin{tabular}{llcc} 
& & \multicolumn{2}{c}{$\mathrm{IC}_{50}(\mu \mathrm{M})$} \\
\cline { 3 - 4 } no. & \multicolumn{1}{c}{$\mathrm{R}$} & $2.5 \pm 0.3$ & strand transfer \\
$\mathbf{4 a} \mathbf{a}^{b}$ & $\mathrm{H}$ & $4.3 \pm 0.6$ & $0.019 \pm 0.002$ \\
$\mathbf{4 b}$ & $\left(\mathrm{CH}_{2}\right)_{3} \mathrm{OH}$ & $8.2 \pm 1.2$ & $0.0027 \pm 0.0004$ \\
$\mathbf{4 c}$ & $\left(\mathrm{CH}_{2}\right)_{5} \mathrm{OH}$ & $19 \pm 1$ & $0.00059 \pm 0.00011$ \\
$\mathbf{4 d}$ & $\left(\mathrm{CH}_{2}\right)_{6} \mathrm{OH}$ & $32 \pm 6$ & $0.0054 \pm 0.0008$ \\
$\mathbf{4 e}$ & $\left(\mathrm{CH}_{2}\right)_{8} \mathrm{OH}$ & & $0.0037 \pm 0.0013$
\end{tabular}

${ }^{a}$ Assays were performed using a gel-based protocol with $\mathrm{Mg}^{2+}$ cofactor as described. ${ }^{43}{ }^{b}$ Previously reported values. ${ }^{24}$

equivalent to $\mathrm{RAL}\left(\mathrm{EC}_{50} \simeq 1 \mathrm{nM}\right)$ against $\mathrm{HIV}$ vectors carrying wild-type (WT) IN in single-round replication assays (Table 2). In similar assays, RAL shows a significant loss of antiviral efficacy when the HIV-1 vectors employed carried the drug resistance mutations Y143R, $\mathrm{N} 155 \mathrm{H}$, or $\mathrm{Q} 148 \mathrm{H} / \mathrm{G} 140 \mathrm{~S}$ mutants $\left(\mathrm{EC}_{50} \simeq 160 \mathrm{nM}, 150 \mathrm{nM}\right.$, and $1900 \mathrm{nM}$, respectively). In contrast, 4a shows effective retention of antiviral potency against both the $\mathrm{Y} 143 \mathrm{R}$ and $\mathrm{N} 155 \mathrm{H}$ variants $\left(\mathrm{EC}_{50} \simeq 2 \mathrm{nM}\right.$ and $5 \mathrm{nM}$, respectively, Table 2). ${ }^{24}$ These values are similar to those displayed by DTG; however, DTG is more potent than 4a against the $\mathrm{Q} 148 \mathrm{H} / \mathrm{G} 140 \mathrm{~S}$ double mutant $\left(4 \mathrm{a} \mathrm{EC}_{50} \simeq 35 \mathrm{nM}\right.$; DTG $\left.\mathrm{EC}_{50} \simeq 6 \mathrm{nM}\right) .^{24}$

Overcoming Loss of Potency against the Integrase Double Mutant Q148H/G140S. The extended tricyclic ring system of DTG permits its third ring (highlighted in green in Figure 1) to contact the $\beta 4-\alpha 2$ loop in the active site of IN (highlighted in orange, Figure 2). Maintenance of interactions in this region has been suggested to be important for the binding of DTG, as well as for the binding of other second generation INSTIs. $^{20,29-31}$ In order to determine the importance of these interactions, we modified $\mathbf{4 a}$ by adding substituents at its 6-position (highlighted in green in Figure 1). Our intent was to create hydrogen bonds with the protein or with bound water molecules proximal to the 6-position. Although there are a variety of constructs that could be used, the work reported herein is focused on simple linear methylene chains of increasing lengths with terminal hydroxyl groups (compounds $\mathbf{4 b} \mathbf{b} \mathbf{4 e}$, Table 1 and Supporting Information Scheme S1). The in vitro ST inhibitory potencies were relatively independent of chain length in going from three methylenes $\left(4 \mathbf{b}, \mathrm{IC}_{50}\right.$ value $\left.=11 \mathrm{nM}\right)$ to eight methylenes $\left(4 \mathrm{e}, \mathrm{IC}_{50}\right.$ value $=$ $5 \mathrm{nM})$. The most potent analogue (by a small margin) was $4 \mathrm{c}$ with $\mathrm{IC}_{50}=2.7 \mathrm{nM}$; Table 1), which also displayed an antiviral inhibitory potency against the Q148H/G140S mutant (4c, $\mathrm{EC}_{50} \simeq 7 \mathrm{nM}$ ), which was similar to that of DTG (Table 2). 
Table 2. Antiviral Potencies in Cells Infected with HIV-1 Vectors That Carry WT or Resistant IN Mutants

\begin{tabular}{|c|c|c|c|c|c|c|}
\hline \multirow[b]{2}{*}{ no. } & \multirow[b]{2}{*}{$\mathrm{CC}_{50}(\mu \mathrm{M})^{a}$} & \multirow[b]{2}{*}{$\mathrm{EC}_{50}(\mathrm{nM})^{b}$} & \multicolumn{4}{|c|}{$\mathrm{EC}_{50}(\mathrm{nM})^{c, d}$} \\
\hline & & & Y143R & $\mathrm{N} 155 \mathrm{H}$ & G140S/Q148H & G118R \\
\hline $1^{e}$ & $>250$ & $4 \pm 2$ & $162 \pm 16(41)$ & $154 \pm 33(39)$ & $1900 \pm 300(475)$ & $36 \pm 5(9)^{e}$ \\
\hline 2 & $>250$ & $6.4 \pm 0.8$ & $7.9 \pm 2.3(1.2)$ & $90 \pm 18(14)$ & $5700 \pm 1100(891)$ & $21 \pm 10(3.3)$ \\
\hline $3^{e}$ & $>250^{e}$ & $1.6 \pm 0.9^{e}$ & $4.3 \pm 1.2(2.7)^{e}$ & $3.6 \pm 1.3(2.3)^{e}$ & $5.8 \pm 0.5(3.6)^{e}$ & $13 \pm 5(8.1)^{e}$ \\
\hline $4 a^{e}$ & $>250^{e}$ & $1.1 \pm 0.7^{e}$ & $2.5 \pm 0.6(2.3)^{e}$ & $5.3 \pm 2.3(4.8)^{e}$ & $35 \pm 9(32)^{e}$ & $16 \pm 5(15)^{e}$ \\
\hline $4 b$ & $>250$ & $3.1 \pm 2.0$ & $2.8 \pm 0.9(0.90)$ & $8.5 \pm 3.1(2.7)$ & $13.4 \pm 6.5(4.3)$ & $10 \pm 1.6(3.2)$ \\
\hline $4 c$ & $>250$ & $1.3 \pm 0.2$ & $3.0 \pm 0.5(2.3)$ & $2.4 \pm 0.8(1.8)$ & $6.9 \pm 2.3(5.3)$ & $5.3 \pm 1.6(4.1)$ \\
\hline $4 d$ & $>250$ & $2.3 \pm 0.6$ & $2.1 \pm 1.4(0.91)$ & $2.7 \pm 1.0(1.2)$ & $9.4 \pm 3.6(4.1)$ & $6.4 \pm 2.5(2.8)$ \\
\hline $4 e$ & $>250$ & $3.7 \pm 0.4$ & $3.2 \pm 0.8(0.86)$ & $6.5 \pm 2.7(1.8)$ & $7.7 \pm 1.9(2.1)$ & $11 \pm 1(3.0)$ \\
\hline \multirow[t]{2}{*}{ 4f } & $>250$ & $2 \pm 0.1$ & $0.6 \pm 0.1(0.3)$ & $2.1 \pm 1.2(1)$ & $5.2 \pm 0.3$ & $11.4 \pm 3.5(6)$ \\
\hline & \multicolumn{6}{|c|}{$\mathrm{EC}_{50}(\mathrm{nM})^{c, d}$} \\
\hline no. & \multicolumn{2}{|c|}{$\mathrm{R} 263 \mathrm{~K}$} & T66I & E92Q & H51Y & H51Y/R263K \\
\hline $1^{e}$ & \multicolumn{2}{|c|}{$5.7 \pm 2.3(1.4)^{e}$} & $2.8 \pm 0.4(0.7)$ & $30 \pm 10(7.5)$ & $3.4 \pm 0.2(0.2)$ & $6 \pm 2.3(1.5)$ \\
\hline 2 & \multicolumn{2}{|c|}{$10 \pm 6(1.6)$} & $66 \pm 1(10)$ & $154 \pm 34(24)$ & $4.5 \pm 2.1(0.7)$ & $53 \pm 18(8)$ \\
\hline $3^{e}$ & \multicolumn{2}{|c|}{$11 \pm 3(6.9)^{e}$} & $0.9 \pm 0.8(0.56)$ & $2.3 \pm 0.4(1.4)$ & $3.2 \pm 0.2(2)$ & $16 \pm 2(10)$ \\
\hline $4 a^{e}$ & \multicolumn{2}{|c|}{$6.4 \pm 2.3(5.8)^{e}$} & $0.6 \pm 0.3(0.54)$ & $3.0 \pm 1.8(2.7)$ & $1.0 \pm 0.6(0.91)$ & $3.2 \pm 0.9(2.9)$ \\
\hline $4 b$ & \multicolumn{2}{|c|}{$2.5 \pm 0.8(0.81)$} & $1.1 \pm 0.1(0.35)$ & $4.8 \pm 2.0(1.5)$ & $2.6 \pm 0.5(0.84)$ & $15 \pm 2(4.8)$ \\
\hline $4 c$ & \multicolumn{2}{|c|}{$2.6 \pm 0.1(2.0)$} & $0.93 \pm 0.24(0.72)$ & $3.8 \pm 2.3(2.3)$ & $3.8 \pm 0.6(2.9)$ & $2.6 \pm 1.4(2.0)$ \\
\hline $4 d$ & \multicolumn{2}{|c|}{$8.4 \pm 2.3$} & $0.50 \pm 0.35(0.22)$ & $0.74 \pm 0.13(0.32)$ & $1.6 \pm 0.1(0.70)$ & $5.2 \pm 1.6(2.3)$ \\
\hline $4 e$ & \multicolumn{2}{|c|}{$3.8 \pm 1.1(1.0)$} & $1.7 \pm 0.5(0.46)$ & $2.6 \pm 0.2(0.70)$ & $4.8 \pm 1.1(1.3)$ & $22 \pm 8(5.9)$ \\
\hline 4f & \multicolumn{2}{|c|}{$5.3 \pm 0.8(2)$} & $0.7 \pm 0.1(0.3)$ & $1.2 \pm 0.6(0.6)$ & $0.8 \pm 0.5(0.4)$ & $5.3 \pm 0.4$ \\
\hline
\end{tabular}

${ }^{a}$ Cytotoxic concentration resulting in 50\% reduction in the level of ATP in human osteosarcoma (HOS) cells. ${ }^{b}$ Values obtained from cells infected with a lentiviral vector carrying WT IN. ${ }^{c}$ Cells were infected with viral vectors carrying IN mutations. The table shows the $\mathrm{EC}_{50}$ values. ${ }^{d}$ Fold-change (FC) in $\mathrm{EC}_{50}$ relative to WT. ${ }^{e}$ Previously reported values. ${ }^{24}$

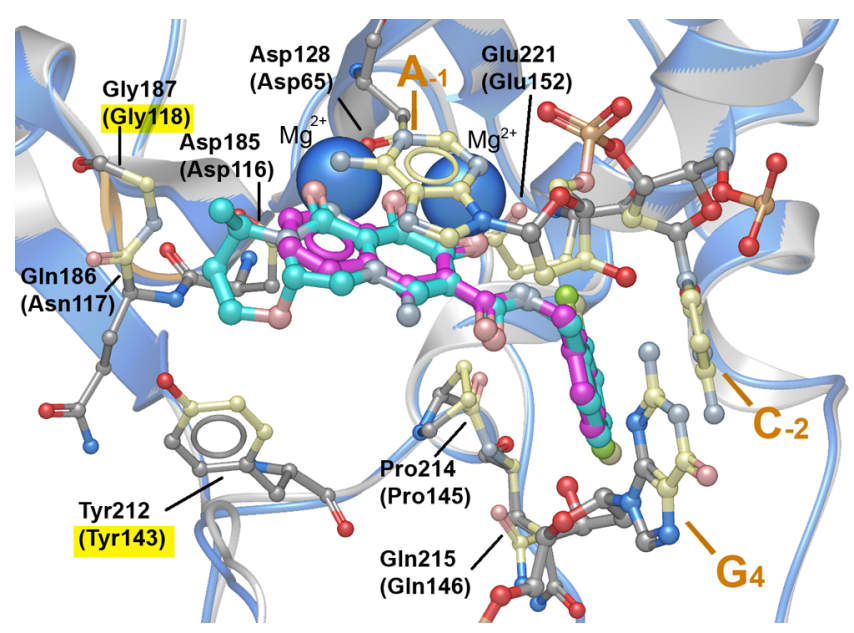

Figure 2. DTG and 4a binding in the active site of the PFV intasome. PFV-bound DTG (cyan with protein ribbon in gray) is shown with $\mathrm{Mg}^{2+}$ ions (solid blue spheres) along with the A-1 and C-2 nucleotides of 3 -processed viral DNA and the G4 nucleotide of the uncleaved complementary strand (PDB code $3 S 3 \mathrm{M}$ ). Interacting residues are shown with contacting carbons shown in cream (corresponding IN residues are indicated in parentheses). The protein $\beta 4-\alpha 2$ loop is highlighted in orange. Contact residues associated with RAL resistance (Y143R and G118R) are highlighted in yellow. PFV-bound $\mathbf{4 a}$ is superimposed onto the DTG-bound structure with the ligand carbons in magenta and the protein ribbon in blue.

Compounds with 6-Substituents Potently Inhibit DTG-resistant IN Mutants. We expanded the panel of HIV vectors used in the antiviral assays to include additional IN mutants that have a reduced susceptibility to DTG, including the G118R, R263K, T66I, E92Q, H51Y single mutants and the H51Y/R263K double mutant. ${ }^{13-16}$ We were particularly interested in whether $4 c$ retained potency against these resistant mutants, because $4 \mathrm{c}$ had the best profile against the original panel of mutants. This compound inhibited the replication of the T66I, E92Q and H51Y mutants as well as DTG and, encouragingly, also exhibited improved antiviral potencies relative to DTG against the G118R and R263K single mutants and the H51Y/R263K double mutant (Table 2). Importantly, none of the analogues of $\mathbf{4}$ showed appreciable cytotoxicity.

X-ray Crystal Structures of $4 a$ and $4 c$ Bound to the PFV Intasome. We refined a structure of the PFV intasome in complex with $4 \mathrm{a}$ to a resolution of $2.58 \AA$ and compared it to the previously reported structure with DTG (PDB accession code: $3 \mathrm{~S} 3 \mathrm{M}) .{ }^{20}$ The 8 -naphthyridine nitrogen and $N$-hydroxyl of $4 \mathbf{a}$ correspond to the 6-oxo and 7-hydroxyl heteroatoms of DTG, and the naphthyridine 2-oxo carbonyl of 4a corresponds to the ring 8-oxo carbonyl of DTG (Figure 2). As we had anticipated in our original design strategy, ${ }^{23}$ the halobenzyl carboxamido group of $\mathbf{4 a}$ is not involved in metal chelation. We also examined the contact residues for DTG (shown in Figure 2 and listed in the Supporting Information Table S2) and noted (as has previously been reported ${ }^{20}$ ) that DTG makes contact with PFV integrase Gly187 and Tyr212, which correspond to HIV-1 IN residues Gly118 and Tyr143, which are associated with RAL-resistant mutations (Table 2). In contrast, 4a does not make contact with either the Gly187 or Tyr212 residues (see Supporting Information Table S2). The 15-fold loss of potency of the G118R virus to $4 a$ is likely due to steric exclusion of the compound by the bulky Arg side chain. Neither DTG nor 4a directly contacts other IN residues associated with INSTI resistance. This is consistent with prior observations that for inhibitors binding to the IN active site, resistance mutations do not usually arise due to a loss of IN-drug interactions. ${ }^{19,20}$

Next, we determined the structure of the PFV intasome crystallized with the 6-substituted compound 4c (Figure 3). 
A.

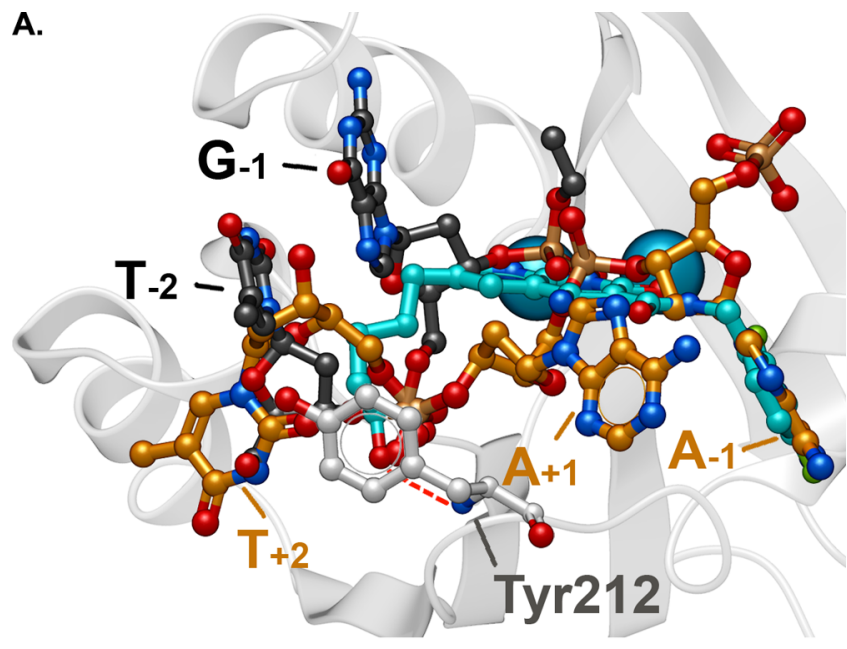

B.

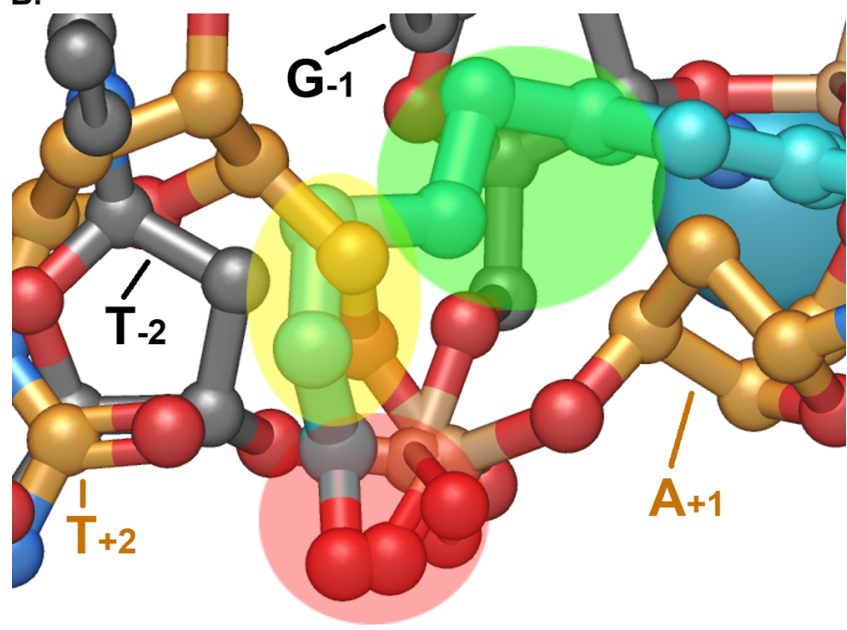

Figure 3. Compound $4 \mathrm{c}$ in the active site of the PFV intasome. (A) Superposition of the binding of $4 c$ (blue) with pre- 3 '-processed DNA (carbons in gold; PDB code: $4 \mathrm{E}^{2} \mathrm{I}^{25}$ ) and target DNA (carbons in black; PDB code: $4 \mathrm{E} 7 \mathrm{~K}^{25}$ ). The bases of proximal nucleotides of the viral and host DNAs are annotated. The hydrogen bond between the 6- substituent terminal hydroxyl of $4 \mathrm{c}$ with the backbone amide nitrogen of Tyr212 is shown. (B) Closeup of panel A highlighting regions of correspondence between the 6-side chain of $4 \mathrm{c}$ and the nucleotides of the substrate DNAs: red, phosphoryl oxygens; yellow, $\mathrm{T}_{+2}$ deoxysugar; and green, $\mathrm{G}_{-1}$ deoxysugar. The IN residue Tyr212 is not shown for clarity.

The structure revealed that the hydroxyl group of the pentan-5ol side chain of $\mathbf{4 c}$ forms a hydrogen bond with the backbone amide of PFV IN Tyr212, a residue that corresponds to HIV-1 IN Tyr143 (Figure 3A). Of particular interest, this same amide group interacts with the phosphoryl groups of both the viral and target DNAs during the $3^{\prime}-\mathrm{P}$ and ST reactions, respectively. ${ }^{25}$ Until now, RAL was the only INSTI known to make interactions with the backbone amide of Tyr143. However, in case of RAL, the interaction depends on the stacking of its 1,3,4-oxadiazole ring with the side chain of Tyr143, making the drug highly susceptible to the Y143R mutation. ${ }^{18,19,32}$

By comparing the crystal structure of the PFV intasome with $4 \mathrm{c}$ bound to the structure of the PFV intasome with viral DNA bound prior to 3'-P (PDB code 4E7I), we discovered that the position of the terminal portion of the 6-side chain of $4 \mathrm{c}$ coincides with parts of the $T_{+2}$ deoxyribose sugar of the unprocessed viral DNA. We also compared the $4 \mathrm{c}$ bound structure with the target capture complex, which has both the $3^{\prime}$-P viral DNA and the host DNA held in orientations suitable for insertion of the viral DNA into the host DNA (PDB code $4 \mathrm{E} 7 \mathrm{~K})$. The naphthyridine-proximal region of the 6-side chain of $4 \mathrm{c}$ overlaps with a portion of the deoxyribose sugar of the $\mathrm{G}_{-1}$ nucleotide in the target DNA structure (Figure 3B). Thus, there is overlap of the 6-side chain of $4 \mathrm{c}$ with both unprocessed viral DNA in a pre- $3^{\prime}-\mathrm{P}$ complex and the target DNA in a ST complex. It is noteworthy that the entire series of compounds $\mathbf{4 b}-\mathbf{4 e}$ had similar antiviral potencies against the panel of resistant IN mutants shown in Table 2. This is consistent with several of the linear polymethylene 6-substituents maintaining key interactions through conformational flexibility.

Interactions of the Intasome with a Sulfonylcontaining 6-Substituent. On the basis of the structural results discussed above, we introduced a sulfonyl functionality within the 6-substituent to yield compound 4f. This was done in an attempt to make $\mathbf{4 f}$ mimic the interactions IN shows with its DNA substrate phosphatediesters. Accordingly, 4f showed potencies equal to or slightly better than DTG in cell-based antiviral assays against a panel of viral vectors that included the major RAL and DTG-resistant IN mutants (Table 2).

To visualize the interactions $4 \mathbf{f}$ makes with the active site of IN, we determined a crystal structure of $\mathbf{4} f$ bound in the active site of the PFV intasome (Figure 4). The structure revealed that one of the sulfonyl oxygens of $\mathbf{4 f}$ forms hydrogen bonds with the main chain amide and the phenolic side chain of Tyr212 via bridging water molecules, while the other makes close van der Waals contacts with backbone atoms of Gln 186 and Gly187 in the $\beta 4-\alpha 2$ loop (Figure 4A). Strikingly, the sulfonylphenyl ring is bent back under the plane of the metal-chelating naphthyridinone ring system, where it makes an intramolecular $\pi-\pi$ stacking interaction ( $3.3 \AA$ separation) (Figure $4 \mathrm{~A}$ and B). By superimposing this structure with the structures of both the uncleaved (pre-3'-P) viral DNA and the target DNA (ST) substrates (PBD codes 4E7I and 4E7K, respectively), ${ }^{25}$ it is apparent that the sulfonyl group fills the envelope formed by the overlap in the positions of both the bound uncleaved viral and the bound target DNAs (Figure 4B). In addition, the position of the sulfonylphenyl ring is similar to the position of the deoxyribose sugar of the adenosine $\mathrm{A}_{-1}$ in the scissile A-T dinucleotide of the uncleaved viral DNA, while other portions of the 6-side chain are similar to the position of the deoxyribose sugar of the $G_{-1}$ guanosine in the target DNA (Figure $4 B$ ).

The face-on-face stacking of the sulfonylphenyl ring of $\mathbf{4 f}$ onto its own heterobicyclic core allows it to almost completely fill the catalytic pocket. When $\mathbf{4 f}$ is bound, its sulfonylphenyl ring packs against the side chain $\beta$-methylene of the metalchelating residue D185 $(\simeq 3.8 \AA)$ and against the $\alpha$-methylene of the Tyr212 backbone $(\simeq 3.7 \AA)$. The sulfonylphenyl ring also nestles against the back face of the Pro214 pyrrolidine ring, a key residue that helps form the hydrophobic binding pocket where the halobenzyl moiety is bound (Figure 4A). These results demonstrate improved overlap in the bound form of $\mathbf{4 f}$ and portions of both target and viral DNA substrates as compared to the bound form of $\mathbf{4 c}$, which was our goal in designing $4 \mathbf{f}$.

INSTIs are among the most effective antiretroviral drugs for the treatment of HIV/AIDS. However, as has been true for all anti-HIV drugs, the development of resistant IN mutants has been a limitation for RAL and EVG, the first two of the three 
A.

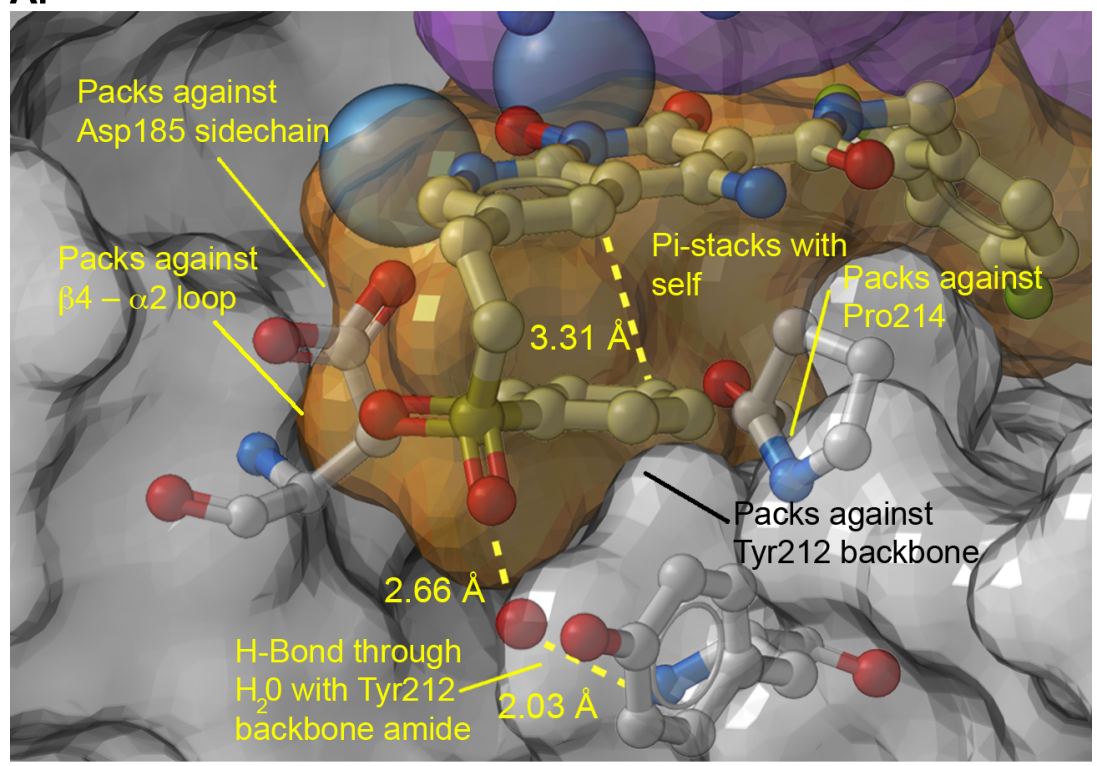

B.

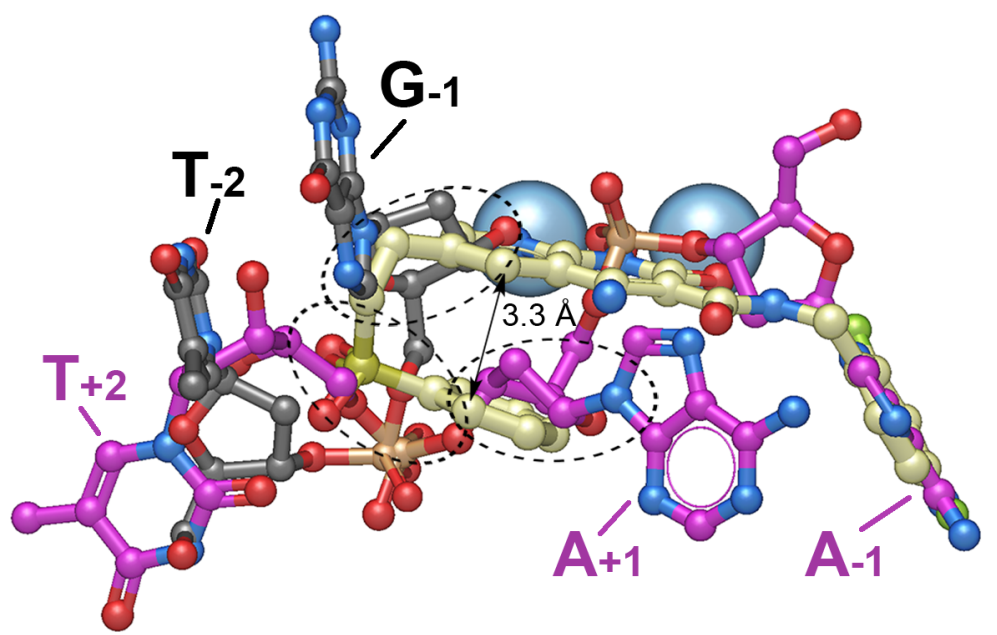

Figure 4. PFV Intasome crystal structure complexed with 4f. (A) Crystal structure oriented to show the complexed $\mathrm{Mg}^{2+}$ ions (blue spheres) and the semitransparent surface of $\mathbf{4 f}$ (orange surface with carbons in cream), $3^{\prime} \mathrm{P}$ DNA (purple surface), and protein (gray surface) with key protein residues shown (carbons in light gray). Important binding interactions are indicated. (B) Bound $\mathbf{4 f}$ with superimposed pre- $3^{\prime}$-processed DNA (carbons in purple; PDB code: $4 \mathrm{E}^{25}$ ) and target DNA (carbons in black; PDB code: $4 \mathrm{E} 7 \mathrm{~K}^{25}$ ). Regions where the side chain of $4 \mathrm{f}$ overlaps with DNA are indicated by black dashed ovals.

current FDA-approved members of this family. Although the most recently introduced INSTI, DTG, appears to be considerably more resilient to viral resistance, it is not infallible. Therefore, continued research is needed to obtain INSTIs that are active against all of the known resistant mutants.

In the current work, we introduced functionality at the 6position of our previously reported INSTI 4 a to take advantage of potential binding interactions afforded by the third $[(R)-4$ methyl-1,3-oxazinane] ring of DTG. Although the approach was initially empirical, it yielded analogues, such as $\mathbf{4 c}$, that retain excellent inhibitory potencies against the major drugresistant IN mutants. By analyzing crystal structures of the PFV intasome in complex with some of the new compounds, we realized that the 6-substitutent of $4 \mathrm{c}$ recapitulates functionalities present in both an uncleaved viral DNA substrate in the pre-3'-P complex and the target DNA prior to ST. This represents an unusual form of "bi-substrate" mimicry, which we attempted to enhance by introducing a sulfone group at the 6position. Among the resulting compounds is the phenylsulfone 4f, which shows potent activity against recombinant HIV IN in biochemical assays and good efficacy in assays done in cell culture against a broad panel of viral mutants carrying the major RAL and DTG IN resistance mutations. Importantly, a crystal structure of $\mathbf{4} \mathbf{f}$ within the PFV intasome shows that $\mathbf{4 f}$ occupies the sites where both the target and viral DNA are bound and largely fills the space that is available at the catalytic center.

The molecular interactions of $4 \mathrm{c}$ and, to a greater extent, $4 \mathrm{f}$ exemplify a more general concept of "substrate envelope" originally used to explain why some HIV protease inhibitors are able to retain efficacy against multiple resistance mutations. ${ }^{33}$ The rationale underlying this principle is that any mutant form of an enzyme that retains good enzymatic activity must be able to bind its cognate substrate(s). Thus, an inhibitor that binds to an enzyme in a fashion such that the inhibitor remains entirely 
within the envelope defined by the structure of the normal substrate(s) will be able to bind efficiently to both WT and (enzymatically active) mutant forms of the enzyme. The fact that our 6-substituted analogues have structural components that bind in ways that are similar to the target and viral DNAs points the way to developing new and more broadly effective INSTIs. $^{25}$

Shape complementarity also plays an important role in protein-ligand recognition. ${ }^{34}$ While simultaneously remaining with an envelope defined by the cognate substrates, the 6substituted analogues, $\mathbf{4 c}$ and $\mathbf{4 f}$, occupy more of the catalyticsite volume (as defined by the structure of the protein and the 3 '-processed DNA) than the unsubstituted parent $\mathbf{4 a}$. Thus, the compounds have good shape complementarity without protruding beyond the substrate envelope. This has resulted in potent inhibitors that retain high efficacy against the entire panel of resistant mutant forms of IN.

Our findings provide a unifying theme for understanding why there are common structural features in some of the most successful second generation INTSIs. For example, although it is known that the terminal $(R)$-4-methyl-1,3-oxazinane ring of DTG is important for its potency against resistant mutant forms of IN, this portion of the molecule was developed empirically. ${ }^{30}$ In the PFV cocrystal structures of DTG (PDB code: $3 \mathrm{~S} 3 \mathrm{M}){ }^{20}$ the position where the third ring is bound overlaps with portions of a bound MK-2048 molecule (PDB code: $3 \mathrm{OYB}) .{ }^{19} \mathrm{MK}-2048$ is another second generation tricyclic INSTI that retains good inhibitory potency against RALresistant mutants. For both DTG and MK-2048, the overlapping regions project toward the $\beta 4-\alpha 2$ loop in the respective PFV intasome cocrystal structures (see Figure 2). Examination of structures of the PFV intasome with other second generation INSTIs bound, including "Compound G" (PDB code: 3OYG) ${ }^{19}$ and MK0536 (PDB code: $3 \mathrm{OYH}{ }^{19}$ these structures are shown in the Supporting Information, Figure S2) and its more recent analogues, ${ }^{31}$ shows that these molecules bind within a volume that is defined by the bound substrates. We previously proposed that the contacts made between the compounds and HIV-1 IN may be an important feature that helps some INSTIs retain efficacy against RAL-resistant mutants. $^{19}$

Our current work shows that portions of the most successful INSTIs can be viewed as occupying regions defined by the binding of the precleaved viral DNA and target DNA, which helps to explain why the compounds are broadly effective against the known drug resistant IN mutants. The principle of maximizing shape complementarity and recapitulating the structural components (structural complementarity) of two of the substrates, the target and viral DNAs, could serve as a guiding principle for the development of new INSTIs.

\section{METHODS}

Synthetic. Detailed synthetic procedures and analytical data for the synthesis of final products $\mathbf{4 b}-\mathbf{4 f}$ are provided in the Supporting Information.

Recombinant Proteins, Biochemical Assays, and Crystallography. IN proteins were produced in E. coli; HIV-1 IN biochemical assays and PFV crystallography were carried out as previously described. ${ }^{18,19,35}$ Detailed experimental protocols are provided in the Supporting Information.

Single-round HIV-1 Infectivity Assay. Assays were performed using the human embryonic kidney cell culture cell line 293 acquired from the American type culture collection (ATCC) and the human osteosarcoma cell line, HOS, obtained from Dr. Richard Schwartz
(Michigan State University, East Lansing, MI) using methods that have been previously reported ${ }^{36,37}$ and are described in detail in the Supporting Information.

X-ray Crystallography. PFV intasome crystals grown as previously described ${ }^{19,20}$ were soaked in the presence of $0.5-1 \mathrm{mM}$ INSTIs in cryoprotection solution prior to snap freezing in liquid nitrogen. X-ray diffraction data were collected on beamline I04 of the Diamond Light Source (Oxfordshire, UK) and processed using XDS ${ }^{38}$ and Scala. ${ }^{39}$ Structures were solved using rigid-body refinement of 4BDZ (protein and DNA components), and small molecules were fitted into difference maps. The structures were built in Coot, ${ }^{40}$ refined in Phenix, ${ }^{41}$ and validated using MolProbity. ${ }^{42}$ Data collection and refinement statistics are given in Supporting Information Table S1.

\section{ASSOCIATED CONTENT}

\section{Supporting Information}

The Supporting Information is available free of charge on the ACS Publications website at DOI: 10.1021/acschembio.5b00948.

Detailed chemical synthesis and compound characterization, details of HIV-1 IN biochemical assays, HIV-1 vector constructs, single-round HIV-1 infectivity assay, and X-ray crystallography data collection and refinement statistics (PDF)

\section{Accession Codes}

Coordinates and structure factors were deposited with the Protein Data Bank under accession codes 5FRM, 5FRN, and SFRO.

\section{AUTHOR INFORMATION}

\section{Corresponding Author}

*Tel: 301-846-5906. Fax: 301-846-6033. E-mail: burkete@ helix.nih.gov.

\section{Notes}

The authors declare the following competing financial interest(s): Compounds described in the paper are included in pending or filed patent applications.

\section{ACKNOWLEDGMENTS}

We thank the staff of the Diamond Light Source beamline I04 for assistance with X-ray data collection. Our studies are supported by the NIH Intramural Program, Center for Cancer Research (ZIA BC 007363, Z01 BC 007333), National Cancer Institute and by grants from the $\mathrm{NIH}$ Intramural AIDS Targeted Antiviral Program (IATAP).

\section{REFERENCES}

(1) Pommier, Y., and Marchand, C. (2012) Interfacial inhibitors: Targeting macromolecular complexes. Nat. Rev. Drug Discovery 11, $25-36$.

(2) Cherepanov, P., Maertens, G. N., and Hare, S. (2011) Structural insights into the retroviral DNA integration apparatus. Curr. Opin. Struct. Biol. 21, 249-256.

(3) Di Santo, R. (2014) Inhibiting the HIV integration process: Past, present, and the future. J. Med. Chem. 57, 539-566.

(4) Abram, M. E., Ferris, A. L., Shao, W., Alvord, W. G., and Hughes, S. H. (2010) Nature, position, and frequency of mutations made in a single cycle of HIV-1 replication. J. Virol. 84, 9864-9878.

(5) Cuevas, J. M., Geller, R., Garijo, R., Lopez-Aldeguer, J., and Sanjuan, R. (2015) Extremely high mutation rate of HIV-1 in vivo. PLoS Biol. 13, e1002251.

(6) Wainberg, M. A., Zaharatos, G. J., and Brenner, B. G. (2011) Development of antiretroviral drug resistance. N. Engl. J. Med. 365, 637-646. 
(7) Garrido, C., Villacian, J., Zahonero, N., Pattery, T., Garcia, F., Gutierrez, F., Caballero, E., Van Houtte, M., Soriano, V., and de Mendoza, C. (2012) Broad phenotypic cross-resistance to elvitegravir in HIV-infected patients failing on raltegravir-containing regimens. Antimicrob. Agents Chemother. 56, 2873-2878.

(8) Geretti, A. M., Armenia, D., and Ceccherini-Silberstein, F. (2012) Emerging patterns and implications of HIV-1 integrase inhibitor resistance. Curr. Opin. Infect. Dis. 25, 677-686.

(9) Quashie, P. K., Mesplede, T., and Wainberg, M. A. (2013) Evolution of HIV integrase resistance mutations. Curr. Opin. Infect. Dis. 26, 43-49.

(10) Ballantyne, A. D., and Perry, C. M. (2013) Dolutegravir: First global approval. Drugs 73, 1627-1637.

(11) Hurt, C. B., Sebastian, J., Hicks, C. B., and Eron, J. J. (2014) Resistance to HIV integrase strand transfer inhibitors among clinical specimens in the United States, 2009-2012. Clin. Infect. Dis. 58, 423431.

(12) McCormack, P. L. (2014) Dolutegravir: A review of its use in the management of HIV-1 infection in adolescents and adults. Drugs $74,1241-1254$.

(13) Mesplede, T., Quashie, P. K., and Wainberg, M. A. (2012) Resistance to HIV integrase inhibitors. Curr. Opin. HIV AIDS 7, 401408.

(14) Wainberg, M. A., Mesplede, T., and Raffi, F. (2013) What if HIV were unable to develop resistance against a new therapeutic agent? BMC Med. 11, 249.

(15) Shah, B. M., Schafer, J. J., and DeSimone, J. A., Jr. (2014) Dolutegravir: A new integrase strand transfer inhibitor for the treatment of HIV. Pharmacotherapy 34, 506-520.

(16) Malet, I., Gimferrer Arriaga, L., Artese, A., Costa, G., Parrotta, L., Alcaro, S., Delelis, O., Tmeizeh, A., Katlama, C., Valantin, M.-A., Ceccherini-Silberstein, F., Calvez, V., and Marcelin, A.-G. (2014) New raltegravir resistance pathways induce broad cross-resistance to all currently used integrase inhibitors. J. Antimicrob. Chemother. 69, 21182122.

(17) de Souza Cavalcanti, J., de Paula Ferreira, J. L., Morenade Souza Guimaraes, P., Vidal, J. E., and de Macedo Brigido, L. F. (2015) High frequency of dolutegravir resistance in patients failing a raltegravircontaining salvage regimen. J. Antimicrob. Chemother. 70, 926-929.

(18) Hare, S., Gupta, S., Valkov, E., Engelman, A., and Cherepanov, P. (2010) Retroviral intasome assembly and inhibition of DNA strand transfer. Nature 464, 232-236.

(19) Hare, S., Vos, A. M., Clayton, R. F., Thuring, J. W., Cummings, M. D., and Cherepanov, P. (2010) Molecular mechanisms of retroviral integrase inhibition and the evolution of viral resistance. Proc. Natl. Acad. Sci. U. S. A. 107, 20057-20062.

(20) Hare, S., Smith, S. J., Metifiot, M., Jaxa-Chamiec, A., Pommier, Y., Hughes, S. H., and Cherepanov, P. (2011) Structural and functional analyses of the second-generation integrase strand transfer inhibitor dolutegravir (S/GSK1349572). Mol. Pharmacol. 80, 565-572.

(21) Menendez-Arias, L. (2013) Molecular basis of human immunodeficiency virus type 1 drug resistance: Overview and recent developments. Antiviral Res. 98, 93-120.

(22) Serrao, E., Odde, S., Ramkumar, K., and Neamati, N. (2009) Raltegravir, elvitegravir, and metoogravir: The birth of "me-too" HIV1 integrase inhibitors. Retrovirology 6, 25.

(23) Zhao, X. Z., Smith, S. J., Metifiot, M., Johnson, B. C., Marchand, C., Pommier, Y., Hughes, S. H., and Burke, T. R., Jr. (2014) Bicyclic 1hydroxy-2-oxo-1,2-dihydropyridine-3-carboxamide-containing HIV-1 integrase inhibitors having high antiviral potency against cells harboring raltegravir-resistant integrase mutants. J. Med. Chem. 57, $1573-1582$.

(24) Zhao, X. Z., Smith, S. J., Metifiot, M., Marchand, C., Boyer, P. L., Pommier, Y., Hughes, S. H., and Burke, T. R., Jr. (2014) 4-Amino-1hydroxy-2-oxo-1,8-naphthyridine-containing compounds having high potency against raltegravir-resistant integrase mutants of HIV-1. $J$. Med. Chem. 57, 5190-5202.
(25) Hare, S., Maertens, G. N., and Cherepanov, P. (2012) 3'Processing and strand transfer catalysed by retroviral integrase in crystallo. EMBO J. 31, 3020-3028.

(26) Johns, B. A., Kawasuji, T., and Velthuisen, E. J. (2013) HIV integrase inhibitors. RSC Drug Discovery Ser. 32, 149-188.

(27) Charpentier, C., Karmochkine, M., Laureillard, D., Tisserand, P., Belec, L., Weiss, L., Si-Mohamed, A., and Piketty, C. (2008) Drug resistance profiles for the HIV integrase gene in patients failing raltegravir salvage therapy. HIV Med. 9, 765-770.

(28) Cooper, D. A., Steigbigel, R. T., Gatell, J. M., Rockstroh, J. K., Katlama, C., Yeni, P., Lazzarin, A., Clotet, B., Kumar, P. N., Eron, J. E., Schechter, M., Markowitz, M., Loutfy, M. R., Lennox, J. L., Zhao, J., Chen, J., Ryan, D. M., Rhodes, R. R., Killar, J. A., Gilde, L. R., Strohmaier, K. M., Meibohm, A. R., Miller, M. D., Hazuda, D. J., Nessly, M. L., DiNubile, M. J., Isaacs, R. D., Teppler, H., and Nguyen, B.-Y. (2008) Subgroup and resistance analyses of raltegravir for resistant HIV-1 infection. N. Engl. J. Med. 359, 355-365.

(29) Kawasuji, T., Johns, B. A., Yoshida, H., Weatherhead, J. G., Akiyama, T., Taishi, T., Taoda, Y., Mikamiyama-Iwata, M., Murai, H., Kiyama, R., Fuji, M., Tanimoto, N., Yoshinaga, T., Seki, T., Kobayashi, M., Sato, A., Garvey, E. P., and Fujiwara, T. (2013) Carbamoyl pyridone HIV-1 integrase inhibitors. 2. Bi- and tricyclic derivatives result in superior antiviral and pharmacokinetic profiles. J. Med. Chem. $56,1124-1135$.

(30) Johns, B. A., Kawasuji, T., Weatherhead, J. G., Taishi, T., Temelkoff, D. P., Yoshida, H., Akiyama, T., Taoda, Y., Murai, H., Kiyama, R., Fuji, M., Tanimoto, N., Jeffrey, J., Foster, S. A., Yoshinaga, T., Seki, T., Kobayashi, M., Sato, A., Johnson, M. N., Garvey, E. P., and Fujiwara, T. (2013) Carbamoyl pyridone HIV-1 integrase Inhibitors 3. A diastereomeric approach to chiral nonracemic tricyclic ring systems and the discovery of dolutegravir (S/GSKi349572) and (S/ GSK1265744). J. Med. Chem. 56, 5901-5916.

(31) Raheem, I. T., Walji, A. M., Klein, D., Sanders, J. M., Powell, D. A., Abeywickrema, P., Barbe, G., Bennet, A., Clas, S. D., Dubost, D., Embrey, M., Grobler, J., Hafey, M. J., Hartingh, T. J., Hazuda, D. J., Miller, M. D., Moore, K. P., Pajkovic, N., Patel, S., Rada, V., Rearden, P., Schreier, J. D., Sisko, J., Steele, T. G., Truchon, J. F., Wai, J., Xu, M., and Coleman, P. J. (2015) Discovery of 2-pyridinone aminals: A prodrug strategy to advance a second generation of HIV-1 Integrase strand transfer inhibitors. J. Med. Chem. 58, 8154-8165.

(32) Metifiot, M., Vandegraaff, N., Maddali, K., Naumova, A., Zhang, X., Rhodes, D., Marchand, C., and Pommier, Y. (2011) Elvitegravir overcomes resistance to raltegravir induced by integrase mutation Y143. AIDS 25, 1175-1178.

(33) Shen, Y., Altman, M. D., Ali, A., Nalam, M. N. L., Cao, H., Rana, T. M., Schiffer, C. A., and Tidor, B. (2013) Testing the substrateenvelope hypothesis with designed pairs of compounds. ACS Chem. Biol. 8, 2433-2441.

(34) Nicholls, A., McGaughey, G. B., Sheridan, R. P., Good, A. C., Warren, G., Mathieu, M., Muchmore, S. W., Brown, S. P., Grant, J. A., Haigh, J. A., Nevins, N., Jain, A. N., and Kelley, B. (2010) Molecular shape and medicinal chemistry: A perspective. J. Med. Chem. 53, 3862-3886.

(35) Metifiot, M., Maddali, K., Johnson, B. C., Hare, S., Smith, S. J., Zhao, X. Z., Marchand, C., Burke, T. R., Jr., Hughes, S. H., Cherepanov, P., and Pommier, Y. (2013) Activities, crystal structures and molecular dynamics of dihydro- $1 \mathrm{H}$-isoindole derivatives, inhibitors of HIV-1 integrase. ACS Chem. Biol. 8, 209-217.

(36) Zhao, X. Z., Maddali, K., Metifiot, M., Smith, S. J., Vu, B. C., Marchand, C., Hughes, S. H., Pommier, Y., and Burke, T. R., Jr. (2012) Bicyclic hydroxy- $1 \mathrm{H}$-pyrrolopyridine-trione containing HIV-1 integrase inhibitors. Chem. Biol. Drug Des. 79, 157-165.

(37) Brachmann, A., Koenig, J., Julius, C., and Feldbruegge, M. (2004) A reverse genetic approach for generating gene replacement mutants in Ustilago maydis. Mol. Genet. Genomics 272, 216-226.

(38) Kabsch, W. (2010) Xds. Acta Crystallogr., Sect. D: Biol. Crystallogr. 66, 125-132. 
(39) Evans, P. (2011) An introduction to data reduction: spacegroup determination, scaling and intensity statistics. Acta Crystallogr., Sect. D: Biol. Crystallogr. 67, 282-292.

(40) Emsley, P., and Cowtan, K. (2004) Coot: model-building tools for molecular graphics. Acta Crystallogr., Sect. D: Biol. Crystallogr. 60, 2126-2132.

(41) Adams, P. D., Afonine, P. V., Bunkóczi, G., Chen, V. B., Echols, N., Headd, J. J., Hung, L.-W., Jain, S., Kapral, G. J., Grosse Kunstleve, R. W., McCoy, A. J., Moriarty, N. W., Oeffner, R. D., Read, R. J., Richardson, D. C., Richardson, J. S., Terwilliger, T. C., and Zwart, P. H. (2011) The Phenix software for automated determination of macromolecular structures. Methods 55, 94-106.

(42) Chen, V. B., Arendall, W. B., III, Headd, J. J., Keedy, D. A., Immormino, R. M., Kapral, G. J., Murray, L. W., Richardson, J. S., and Richardson, D. C. (2010) MolProbity: All-atom structure validation for macromolecular crystallography. Acta Crystallogr., Sect. D: Biol. Crystallogr. 66, 12-21.

(43) Zhao, X. Z., Semenova, E. A., Vu, B. C., Maddali, K., Marchand, C., Hughes, S. H., Pommier, Y., and Burke, T. R. (2008) 2,3-Dihydro6,7-dihydroxy- $1 H$-isoindol-1-one-Based HIV-1 Integrase Inhibitors. J. Med. Chem. 51, 251-259. 